\title{
VALORACIÓN Y CUANTIFICACIÓN DE METALES PESADOS EN CARNE DE CERDO, PESCADO, POLLO Y RES COMERCIALIZADOS EN PAMPLONA NORTE DE SANTANDER
}

\section{ASSESSMENT OF HEAVY METALS AND CUANTIFICASION IN PORK, FISH, CHICKEN AND RES MARKET IN PAMPLONA NORTE DE SANTANDER}

\author{
${ }^{*}$ Arrieta $\mathrm{A}^{1}$. Corredor $\mathrm{W}^{2}$. Vera $\mathrm{J}^{3}$. \\ ${ }^{1}$ Universidad de Pamplona, Facultad de Ingenierías y Arquitectura. Maestría en Ciencia y Tecnología de los \\ Alimentos. Km. 1 Vía Bucaramanga, Pamplona Norte de Santander-Colombia. \\ *Correo electrónico: a_0411@hotmail.com \\ ${ }^{2}$ Universidad Francisco de Paula Santander. Facultad de Ciencias Agrarias y Medio Ambiente Cúcuta. \\ ${ }^{3}$ Servicio Nacional de Aprendizaje SENA. Floridablanca Santander. Bucaramanga Norte de Santander- \\ Colombia
}

Recibido 21 de Julio 2015; aceptado 27 de octubre de 2015

\section{RESUMEN}

La contaminación por metales pesados en los alimentos es uno de los temas de mayor importancia a nivel mundial, estos metales no son biodegradables $y$ tienden a bioacumularse en el musculo y en las vísceras de los animales lo que constituye un riesgo para la salud, cuando consumimos estos sub productos adquirimos el metal que el animal ha acumulado y se establece una cadena acumulativa que eleva peligrosamente su concentración. En el presente estudio se valoró la presencia de trazas de Cd, $\mathrm{Cu}, \mathrm{Mo}, \mathrm{Pb}$ y $\mathrm{Zn}$ en carne bovina, porcina, pollo y pescado comercializados en 12 establecimientos en la plaza de mercado de Pamplona Norte de Santander, los metales fueron cuantificados por espectroscopia de absorción atómica, utilizando lámparas de cátodo hueco, un quemador aire-acetileno, se realizaron análisis de correlación de Pearson entre los contenidos de Cada metal. En el caso del Cadmio las concentraciones para este metal superaron los 
Autor a quien dirigirse la correspondencia. *Arrieta Alexander ${ }^{1}$. Correo electrónico a_0411@hotmail.com límites permisibles < 0,050 mg/kg. La carne de pescado obtuvo la mayor concentración de este metal $0,117 \mathrm{mg} / \mathrm{kg}$, seguido de la carne de res con 0,109 mg/kg, mientras que la carne de cerdo obtuvo 0,095 mg/kg y por último la carne de pollo obtuvo $0,079 \mathrm{mg} / \mathrm{kg}$. El contenido de $\mathrm{Cu}$ en las diferentes matrices arrojo los siguientes resultados; La carne de pescado 0,343 $\mathrm{mg} / \mathrm{kg}$, res 0,306 $\mathrm{mg} / \mathrm{kg}$, cerdo 0,243 $\mathrm{mg} / \mathrm{kg}$, pollo 0,221 $\mathrm{mg} / \mathrm{kg}$. Con respecto a La concentración de $\mathrm{Zn}$, la carne de res presentó mayor contenido $8,35 \mathrm{mg} / \mathrm{kg}$. El Mo no fue detectado en ninguna de las muestras.

Palabras clave: Absorción atómica, Metales pesados, Minerales, Carne, salud pública.

\section{ABSTRACT}

The heavy metal contamination in food is one of the most important issues globally, these metals are not biodegradable and tend to bio accumulate in muscle and viscera of animals which is a health risk, when we consume these sub metal products acquired the animal has amassed a cumulative chain and dangerously increases its concentration is established. In this study the presence of traces of $\mathrm{Cd}, \mathrm{Cu}$, $\mathrm{Mo}, \mathrm{Pb}$ and $\mathrm{Zn}$ in beef, pork, chicken meat is valued and fish sold in 12 stores in the market square of Pamplona Norte de Santander, metals were quantified by spectroscopy atomic absorption, using hollow cathode lamps, an air-acetylene burner Pearson correlation analysis between the content of each metal were performed. In the case of cadmium for this metal concentrations they exceeded the permissible limits $<0.050 \mathrm{mg} / \mathrm{kg}$. The fish meat had the highest concentration of this metal $0,117 \mathrm{mg} / \mathrm{kg}$, followed by beef with $0.109 \mathrm{mg} /$ $\mathrm{kg}$, while pork obtained $0.095 \mathrm{mg} / \mathrm{kg}$ and finally got chicken meat $0.079 \mathrm{mg} / \mathrm{kg}$. The Cu content in the different matrices 
produced the following results; Fish meat $0.343 \mathrm{mg} / \mathrm{kg}$, beef $0.306 \mathrm{mg} / \mathrm{kg}$, pork $0.243 \mathrm{mg} / \mathrm{kg}$, chicken $0.221 \mathrm{mg} / \mathrm{kg}$. With respect to the concentration of $\mathrm{Zn}$, beef had higher content $8,35 \mathrm{mg} / \mathrm{kg}$. Mo was not detected in any samples.

Keywords: Atomic absorption, heavy metals, minerals, meat, public health

\section{INTRODUCCIÓN}

Actualmente, los consumidores se ven sometidos a exposiciones prolongadas de sustancias tóxicas, las cuales comúnmente se encuentran en concentraciones muy bajas en los alimentos. Los metales pesados son elementos químicos que poseen interés ambiental por las repercusiones que éstos tienen con su presencia en los diferentes compartimientos ambientales, los niveles altos de estos metales en las zonas nos permiten determinar el nivel de toxicidad de los mismos, su potencial ingreso y afectación en la cadena trófica; lo anterior está asociado a la posibilidad que tienen varios de estos elementos de bioacumularse (Feria, 2009). La contaminación por metales pesados constituye una de las formas más peligrosas para los ecosistemas, dado que son elementos poco o nada biodegradables, tienden a acumularse en los tejidos de animales y vegetales, y permanecen en ellos por largos períodos, desencadenando procesos de bio magnificación y acciones toxico dinámicas, las cuales generan alteraciones metabólicas, mutaciones y transformaciones anatómicas en las especies animales, incluido el hombre (García y Romero, 2008).

La principal ruta de exposición de la población humana a los metales pesados son los alimentos, dada la difusión en el ambiente y acumulación a través de las cadenas tróficas al ser consumidos y acumulados por los animales domésticos trayendo consigo problemas de salud pública. Dentro de este grupo de contaminantes medioambiental los que se destacan por su toxicidad y distribución son; el mercurio, cadmio y plomo. El arsénico y cobre son elementos esenciales para el metabolismo animal, sin embargo, tanto las especies inorgánicas de arsénico (arsenito y arseniato), como concentraciones elevadas 
de cobre son tóxicas. La presencia de metales pesados en el suelo, agua y aire contribuyen a la contaminación de la cadena alimentaria, por lo que los animales destinados al consumo pueden ser utilizados como indicadores para evaluar la exposición humana a estos contaminantes.

Estudios realizados (Huff et al., 2007) sobre la presencia de metales como el cobre, plomo y zinc encontraron que estos son productores de actividad carcinogénica en animales y posteriormente en humanos, por lo que los componentes de cadmio han sido actualmente clasificados como carcinogénicos por la Agencia Internacional de Investigación de Cáncer. En cuanto a estudios realizados por (Marrugo J. L., 2007) sobre la presencia de estos elementos en fuentes de aguas y peces detectaron los niveles de contaminación por metales pesados en la cuenca del río San Jorge en la ciénaga de Ayapel. Por lo que, para conocer el contacto de la población a distintos contaminantes, es importante realizar estudios que determinen la frecuencia y concentración de residuos tóxicos en distintas fuentes, tanto medios ambientales como dietarías.

En el presente estudio fueron seleccionadas muestras de músculos debido a que son la parte comestible y pueden influir directamente en la salud humana debido a que concentraciones de metales en exceso en estos tejidos son potencialmente grave. El objetivo del presente estudio fue valorar las concentraciones de metales pesados presentes en las carnes (cerdo, pescado, pollo, res) comercializadas en la plaza de mercado de Pamplona Norte de Santander.

\section{MATERIALES Y MÉTODOS}

\section{Muestreo}

Se recolectaron muestras de carne de cerdo, pescado, pollo y de res en 12 expendios en la plaza de mercado de pamplona norte de Santander. Se tomaron 120 gramos por muestra y se sub dividieron en tres sub muestras de $40 \mathrm{~g}$ cada una que se colocarán en bolsas de polipropileno identificados con un código para cada muestra. Para la carne de res y la carne de cerdo las muestras fueron tomadas del musculo derecho, para la carne de pollo se tomaron muestras de la pechuga y para las muestras de pescado se tomó musculo de Mojarra roja (Oreochromis spp). Todas las muestras procedían de establecimientos 
certificados en Buenas Prácticas de Manufactura (BPM) localizados en la región Norte de Santander.

\section{Preparación de la muestra}

Para la obtención de la muestra carne de bovino, se tomaron $40 \mathrm{~g}$ de tejido y se eliminó todo el tejido conectivo y adiposo, se obtuvieron muestras de20, $04 \mathrm{~g}$ para carne res, 20,1919 g para de carne cerdo, 20,1193 g para carne de pollo, 20,3004 g para muestra de pescado. Todas las muestras fueron cortadas en trozos pequeños.

\section{Determinación de humedad y cenizas}

Para la determinación de las cenizas se pesaron inicialmente los crisoles previamente rotulados, y luego se adicionaron aproximadamente los gramos de cada muestra, las muestras fueron llevados a mufla a $105^{\circ} \mathrm{C}$ por 1 hora en un equipo (Vulcan 3-550), luego se realizaron las medidas de peso y los cálculos posteriores según metodología propuesta por (Villar CA., et al 2000).

\section{Curva de calibración}

Para la determinación analítica de las muestras de carne de cerdo, pescado, pollo y res, se realizó de acuerdo a la norma
AOAC 999.1017 a Ed, de la siguiente manera; en la Incineración por secado se tomaron $20,04,20,19,20,11,20,30$ gramos de las respectivas muestras y se colocaron en un crisol, se llevaron a calentamiento durante una hora, produciendo una carbonización parcial, luego se llevó a mufla a $105^{\circ} \mathrm{C}$ durante 2 horas, y culminamos en la mufla a $550{ }^{\circ} \mathrm{C}$ durante 4 horas. La calcinación fue incompleta por lo que se procedió a adicionarle $3 \mathrm{ml}$ de ácido clorhídrico (HCL)3N para disolver las sales, y luego someterlo a calentamiento por unos 10 minutos a fuego lento para disolver la muestra, luego fueron filtradas $y$ transferidas a balón volumétrico de $50 \mathrm{Ml}$. La determinación de los niveles de metales pesados se realizó por Absorción Atómica en un equipo SHIMADZU AA 7000 con llama aire-acetileno para determinar $\mathrm{Cd}, \mathrm{Cu}$, $\mathrm{Pb}$ y $\mathrm{Zn}$ y Óxido nitroso-acetileno para determinar Mo.

\section{Análisis estadístico}

Para los datos obtenidos en la determinación de metales pesados, se realizaron análisis de correlación de Pearson para determinar la significancia de las diferencias, con un nivel de confiabilidad de 0.05 . 


\section{RESULTADOS Y DISCUSIÓN}

La Figura 1. Muestra los resultados obtenidos para el metal Cadmio en las cuatro matrices utilizadas en el estudio. De la gráfica se observa que la carne de pescado obtuvo la mayor concentración de Cd $0,117 \mathrm{mg} / \mathrm{kg}$ superando los límites permisibles para este metal < 0,010 mg/kg para pescados. Todas las muestras analizadas superaron los límites permisibles $<0,050 \mathrm{mg} / \mathrm{kg}$ de Cd para la carne de res, pollo y cerdo.

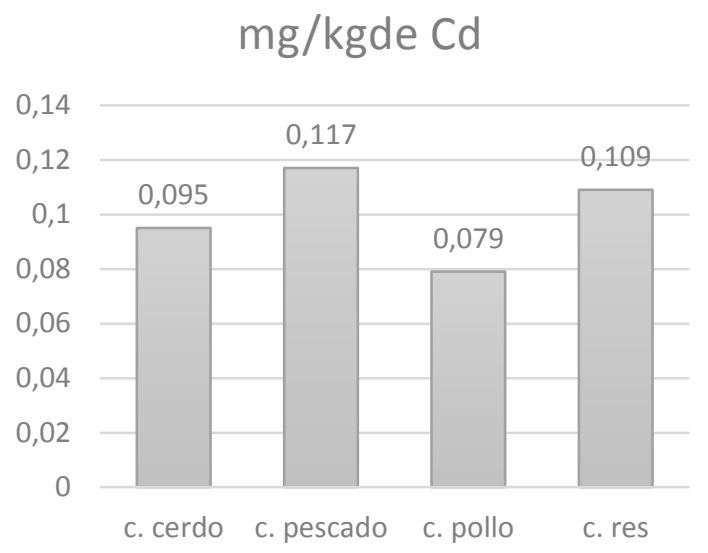

Figura1. Mg/kg Cd. Arieta., et al 2014

De la Figura 2. Se observa que, de las cuatro matrices utilizadas en el estudio, la concentración de $\mathrm{Zn}$ fue mayor en la carne de res ,35 mg / kg superando 0,05mg/kg los imites permisibles para este metal con valores similares a aquellos encontrados en otras investigaciones, los valores en esta investigación son superiores a las reportadas por el USDA (4.66 mg/kg) y Duckett et al., 2008 (8.36 mg/100g), seguido de la carne de pescado que obtuvo una concentración $3,519 \mathrm{mg} / \mathrm{kg}$. La carne de pollo fue la especie que arrojó el menor contenido de $\mathrm{Zn}$, con niveles un poco más bajos que los reportados por el USDA [6] y por el INN, (0.8 mg/k g, para muslo y pechuga, respectivamente).

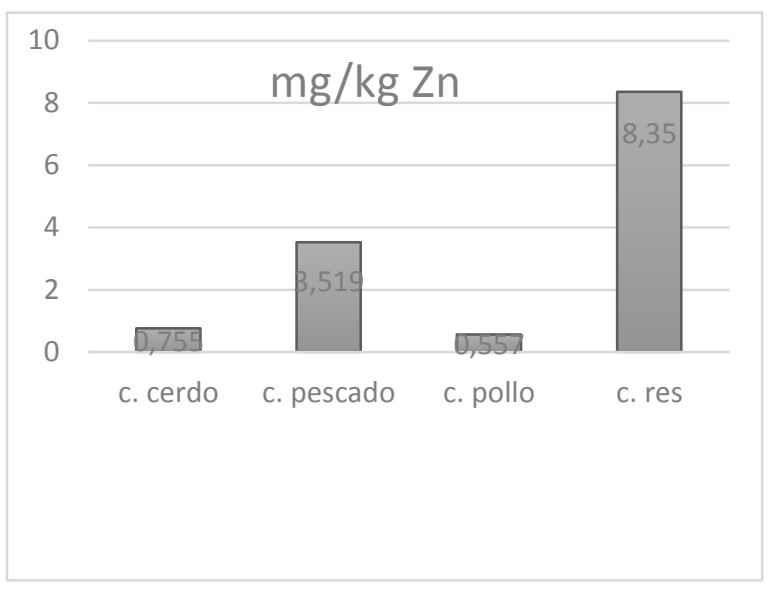

Figura 2. Mg/kg Zn. Arieta., et al 2014

De la Figura 3. Se observa que la carne de pescado presentó una mayor concentración $(0,343) \mathrm{mg} / \mathrm{kg}$ de $\mathrm{Cu}$ que el resto de las carnes analizadas, seguido por la carne de res $(0,306) \mathrm{mg} / \mathrm{kg}$ que supera, por su parte, a la de pollo $(0,221) \mathrm{mg} / \mathrm{kg}$ y a la de cerdo $(0,243) \mathrm{mg} / \mathrm{kg}$. Superando los niveles permisibles. 
@LIMENTECH CIENCIA Y TECNOLOGÍA ALIMENTARIA ISSN 1692-7125. Volumen 13, No. 2, p. 163-171, año 2015 Facultad de Ingenierías y Arquitectura Universidad de Pamplona

\section{$\mathrm{mg} / \mathrm{kg} \mathrm{Cu}$}

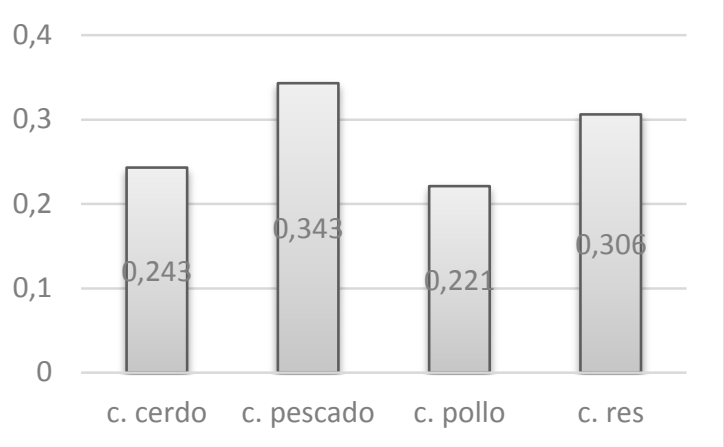

Figura 3. Mg/kg Cu. Arieta., et al 2014.

Es bien conocido que el cobre es un factor limitante clásico para los pescados, ya que es a la vez esencial y tóxico. A medida que las concentraciones superan los límites permisibles, el cobre se convierte en dañino para los pescados pudiendo llegar a ser letal (Villar et. al., 2000). Estudios realizados por Tuzen, et. al., (2003) reportaron niveles medios de plomo en peces comprendidos entre 0,22 a $0,85 \mathrm{mg} \mathrm{kg}$. Por otro lado, Uluozlu., et. al., (2007) investigaron el contenido de plomo en peces se encontrando valores comprendidos entre 0,33 a 0,93 mg para el tejido de los peces comestibles. Mientras que los estudios realizados Bat., et al., (2012) presentaron concentraciones tres veces más altos para $\mathrm{Pb}$ en salmones en comparación con nuestros resultados.

De la Figura 4. Los resultados arrojaron que las muestras de carne de cerdo, pollo, pescado y res se encuentra con los requisitos de inocuidad que se establecen para la carne en cuanto a residuos tóxicos del metal plomo el cual no supero los límites permisibles $<0,10 \mathrm{mg} / \mathrm{kg}$ en carne de pollo, cerdo y res. $Y<0,30 \mathrm{mg} / \mathrm{kg}$ para pescados.

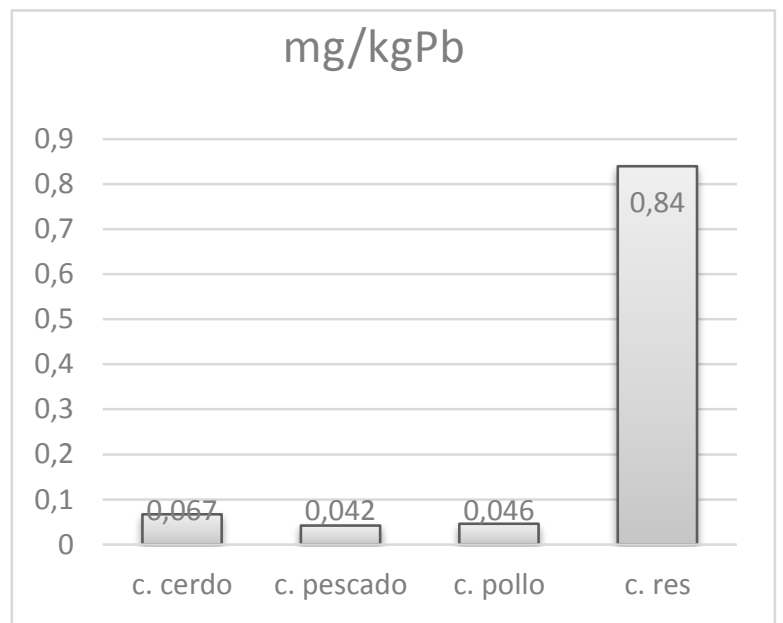

Figura 4. Mg/kg Pb . Arieta., et al., 2014.

\section{CONCLUSIONES}

La primera conclusión del estudio fue que los niveles de metales pesados en las carnes de pollo, cerdo, pescado y de res comercializadas en la plaza de mercado del municipio de Pamplona norte de Santander son preocupantes desde el punto de vista de la salud humana, ya que los valores 
sobrepasaron los límites máximos de residuos fijados en la legislación europea.

Del análisis de los resultados se concluye que las muestras estudiadas cumplieron con los requisitos de inocuidad que se establecen para la carne en cuanto a residuos tóxicos del metal molibdeno y del metal plomo los cuales no fueron detectados en ningunas de las matrices estudiadas.

El molibdeno Mo, no fue detectado en ninguna de las muestras analizadas.

En Colombia, aunque se ha incorporado en la legislación una visión integral de la gestión del Estado que contempla acciones específicas en materia de control de manejo de los residuos, el país muestra deficiencias significativas en materia de manejo de los residuos y sus impactos ambientales, lo cual no permite disminuir los daños resultantes del manejo inadecuado de vertimientos y la disposición de residuos a los cuerpos de agua. Es necesario revisar la legislación y reglamentación existente a nivel nacional en el tema.

Esta información es importante para la elaboración de bases de datos, para asegurar la calidad de los productos cárnicos que se ofrecen al consumidor, así como para dar apoyo al comercio nacional e internacional.

\section{REFERENCIAS BIBLIOGRÁFICAS}

Spiric, A.; Saicic, S. Monitoring chlorinated pesticides and toxic elements in tissues of Food-producing animals in Yugoslavia. Assoc. Off. Anal. Chem. Int. 81(6): 1998.

Kramer, H.L.; Steiner, J.W.; Valiely, P.J. Trace element concentrations in liver, kidney and muscle of Queensland Cattle. Bull. Environ. Contam. Toxicol. 30: 588594. 1983.

EPA. Environmental Protection Agency. Safe wáter standars..NPDWR: U.S.A.
1997: disponible

en

URL:

http://www.epa.gov.

Pietro, J, Gonzales, C, Gutiérrez, R, Contaminacion y fitotoxicidad en plantas por metales pesados provenientes de suelos y agua tropical, subtrop. Agroecosystems. 2009, 10, 29-44

Detection of heavy metals in cattle, in the valleys of the Sinu and San Jorge rivers, department of Cordoba, Colombia. (Madero y Marrugo 2010). 
Universidad Nacional de Colombia, Sede Medellín. Fundación GeoSur. Bogotá, Colombia. (Mancera N \& Álvarez R. 2006).

Riesgo toxicológico en personas expuestas, a suelos y vegetales, con posibles concentraciones de metales pesados, en el sur del atlántico. (Hernández y José 2014).

Composition of Foods: Beef products. RawProcessed-Prepared.

Agriculture Handbook

Number 8-13. United States Department of Agriculture (USDA). Human Nutrition Information Service. Washington D.C., 1990.

Kotula AW and Lusby WR. Mineral composition of muscles of 1 - to 6-yearsold steers. J Anim Sci 1982; 54(3):544548.

Sim DW and Wellington GH. Potassium concentration in bovine muscle as influence by carcass location, breed, sex, energy intake, age and shrunk body weight. J Anim Sci 1976; 42(1):84-91.

Huerta-Leidenz N. El valor nutritivo de la carne de res venezolana vs norteamericana. Memorias de las IV Jornadas del XXX Aniversario de la Escuela de Nutrición: Nutrición y Calidad de Vida. Universidad del Zulia. Facultad de Medicina; 1998. Junio 28- Julio 02:4755. Tabla de Composición de los Alimentos para Uso Práctico. Revisión 1999. Publicación No. 52. Serie de Cuadernos Azules. Venezuela: Instituto Nacional de Nutrición (INN).

Duckett SK, Wagner DG, Yates LD, Dolezal HG \& May SG. Effects of time on feed on beef nutrient composition. J Anim Sci 1993; 71:2079-2088.

Ammerman CB, Loaiza JM, Blue WG, Gamble JF and Martin FG. Mineral composition of tissues from beef cattle under grazing conditions in Panama. $\mathrm{J}$ Anim Sci 1974; 38(1):158-162.

Brito G, Diaz G, Galindo LR, Hardisson A, Santiago Laguna D, García-Montelongo F (1990).Concentration Levels of $\mathrm{Cd}, \mathrm{Pb}$, $\mathrm{Cu}, \mathrm{Zn}, \mathrm{Fe}, \mathrm{Ni}$ and $\mathrm{Mn}$ in canned meat products. Intermetalliccorrelations. Bull Environ Contam Toxicol, 44: 309-316. 